\title{
Intoxicação de vacas leiteiras por farelo de algodão naturalmente contaminado com aflatoxinas
}

\author{
Dairy cows poisoned with cottonseed meal naturally contaminated with aflatoxins
}

\author{
Edlayne Gonçalez ${ }^{1}$ Marina Mori Pinto ${ }^{2}$ Silvio Manginelli ${ }^{3}$ Joana D'arc Felicio $^{4}$
}

\section{RESUMO}

Aflatoxina M1 é um dos metabólitos tóxico da aflatoxina B1, excretada pelo leite de animais que ingerem alimentos contaminados. $O$ presente trabalho descreve o caso de uma fazenda produtora de leite na região de Itú, São Paulo, onde os animais foram alimentados com farelo de algodão naturalmente contaminado com as aflatoxinas. As aflatoxinas B1, B2, G1 e G2 foram identificadas por $C C D e$ quantificadas por fotodensitometria nas concentrações de 43,$5 ; 15,2 ; 9,1$ e 8,6ng. $\mathrm{g}^{-1}$ respectivamente. O leite destes animais foi analisado por cromatografia líquida de alta eficiência (CLAE), e a aflatoxina M1 foi identificada e quantificada $\left(0,64 n g . m L^{-1}\right)$. As concentrações encontradas para as aflatoxinas no farelo de algodão e no leite estão acima dos valores permitidos pela legislação brasileira, representando um risco à saúde pública.

Palavras chave: aflatoxinas, leite, farelo de algodão, contaminação, vacas lactantes.

\section{ABSTRACT}

Aflatoxin $M 1$ is a toxic metabolic from aflatoxin $B 1$, which is excreted in milk after the cows receive contaminated feed. The dairy cows in a farm in Itú city, São Paulo state, Brazil, were fed with cottonseed meal naturally contaminated with aflatoxin. The aflatoxins B1, B2, G1 and G2 were identifiedby TLC and quantified by fotodensitometry and the results were $43.5 ; 15.2 ; 9.1$ and $8.6 \mathrm{ng}^{-g^{-1}}$ respectively. The milk from these cows was also analyzed by higth performance liquid chromatography (HPLC) and 0.64ng. $\mathrm{mL}^{-1}$ aflatoxin M1 was quantified. The aflatoxins concentration were higher than the brazilian low permit, and this should be a damage to public health.

Key words: aflatoxin, milk, cottonseed meal, contamination, dairy cow.

\section{INTRODUÇÃO}

Aspergillus flavus e A parasiticus são fungos de grande importância na agricultura, por contaminar várias culturas e produzirem aflatoxinas, tanto na pré como pós colheita (STEINHART et al., 1996). Dentre as aflatoxinas, destacam-se B1, B2, G1 e G2, que são bem conhecidas e estudadas do ponto de vista toxicológico, (SHARMA \& SALUNKE, 1991). Quando um animal ingere um alimento contaminado com aflatoxina B1, de 0,5 a $5 \%$ da toxina ingerida é biotransformada, no fígado, em aflatoxina M1 (HUSSEIN \& BRASEL, 2001).

Assim, o cuidado com a alimentação animal deve se estender não só ao valor nutricional e econômico, mas também à qualidade do alimento, pois semente de algodão e farelo de algodão são alimentos normalmente utilizados na alimentação de vacas lactantes e, assim como o milho, são muito susceptíveis à contaminação por aflatoxinas (APPLEBAUM et al., 1982). A Organização Mundial de Saúde recomenda a redução do consumo de aflatoxina M1 para um nível que minimize o risco potencial de sua ingestão (LÓPEZ et al., 2001). No Brasil, o limite máximo de aflatoxina M1 permitido segue a definição do MERCOSUL, GMC/RES. n-56/94, onde estipula $0,5 \mu \mathrm{g} . \mathrm{L}^{-1}(\mathrm{ppb})$ em leite fluído e $5,0 \mu \mathrm{g} . \mathrm{L}^{-1}$ (ppb) para leite em pó. Para alimentos destinados ao consumo animal, o limite máximo permitido é 50ng. $\mathrm{g}^{-1}(\mathrm{ppb})$ para a soma das aflatoxinas B1, B2, G1 e G2 (Portaria MA/SNAD/SFA nº 07 de 09/ 11/88).

${ }^{1}$ Pesquisador Científico, Instituto Biológico, Centro de Pesquisa e Desenvolvimento em Sanidade Animal (CPDSA), Av. Cons. Rodrigues Alves, 1252, 04014-002, São Paulo, SP. E-mail: goncalez@biologico.br. Autor para correspondência

${ }^{2}$ Farmacêutico Bioquímico, Instituto Biológico, CPDSA

${ }^{3}$ Zootecnista, Agência Paulista de Tecnologia dos Agronegócios, Av. Miguel Estéfano, 3900, 04301-903, São Paulo, SP.

${ }^{4}$ Pesquisador Científico, Instituto Biológico, CPDSA 
Neste trabalho, avaliou-se a concentração de aflatoxina M1 no leite de vacas lactantes alimentadas com farelo de algodão naturalmente contaminado com aflatoxinas B1, B2, G1 e G2.

\section{RELATO DO CASO}

Fazenda localizada em Itú, SP, produtora de leite, com 40 vacas lactantes pertencentes ao rebanho Jersey, apresentou queda na produção de leite durante seis meses do ano de 2001. O proprietário relatou a morte de 2 novilhas de 8 a 12 meses de idade e de duas vacas de 6 a 7 anos, que apresentaram diarréia intensa; ainda foram relatados sinais clínicos como três abortos e repetição de cios sem causa determinada. Os animais foram alimentados em pastagem e ração $\left(5 \mathrm{~kg}\right.$ ração.dia $\left.{ }^{-1}\right)$ composta por $30 \%$ de farelo de algodão como complemento. Amostra do farelo de algodão foi analisada quanto ao teor de aflatoxinas B1, B2, G1 e G2 e o leite quanto a aflatoxina M1.

\section{Análise do farelo de algodão}

Amostra foi coletada em 05 pontos com diferentes profundidades do lote suspeito e as aflatoxinas analisadas pelo método BF (AOAC 970.45). Neste método, as aflatoxinas são extraídas do substrato com clorofórmio e o extrato reduzido a um volume conhecido. $40 \mu \mathrm{L}$ do extrato e dos padrões de aflatoxinas B1, B2, G1 e $\mathrm{G} 2^{1}$ foram aplicados em placa cromatográfica ${ }^{2}$ e eluída em clorofórmio:acetona (9:1). Para confirmação da presença das aflatoxinas, foi utilizada a cromatografia bidimensional (AOAC 985.17), em que os padrões de aflatoxinas foram aplicados em dois ângulos opostos da placa e a amostra, em um terceiro ângulo, o cromatograma foi desenvolvido com clorofórmio:acetona (9:1) em um sentido. A placa foi então retirada e seca e após um giro de $90^{\circ}$, ela foi desenvolvida em um segundo sentido com éter etílico:metanol:água (96:3:1). As aflatoxinas foram quantificadas por fotodensitometria ${ }^{3}$, comparandose a área do padrão com a da amostra.

\section{Análise do leite} métodos:

A amostra foi analisada através de dois

1. Ensaio imunocromatográfico de interpretação visual, com limite de detecção $0,5 \mathrm{ng} . \mathrm{mL}^{-1}$

A amostra foi centrifugada a $1500 \mathrm{rpm}$ por três minutos, separando-se imediatamente a porção desnatada (inferior) para análise. Foram pipetados $300 \mu \mathrm{L}$ de leite desnatado na tira teste ${ }^{4}$ aquecida a 55 ${ }^{\circ} \mathrm{C}$ por 8 minutos e efetuada a interpretação visual.
2. Cromatografia de imunoafinidade com detecção por Cromatografia Líquida de Alta Eficiência (CLAE)

O leite desnatado $(50 \mathrm{~mL})$ foi aplicado em uma coluna de imunoafinidade para aflatoxina $\mathrm{M}^{5}$. A amostra foi eluída com $1,25 \mathrm{~mL}$ de acetonitrila:metanol (3:2) e 1,25mL de água, coletadas no mesmo frasco. A solução foi agitada e $100 \mu \mathrm{L}$ da amostra foi injetada no cromatográfo ${ }^{6}$ em triplicata, tendo como fase móvel água:acetonitrila:metanol (68:24:8), vazão de 1,0mL. min $^{-1}$ e detector de fluorescência em $360 \mathrm{~nm}$ de excitação e 440nm de emissão (DRAGACCI et al., 2001). O método de quantificação das amostra foi padrão externo, utilizando curva de calibração com 5 pontos $\left(0,161 ; 0,322 ; 0,643 ; 1,287 ; 2,573 n g . \mu \mathrm{L}^{-1}\right)$.

\section{RESULTADOS E DISCUSSÃO}

As aflatoxinas extraídas do farelo de algodão, foram identificadas pela cor da fluorescência que emitiram quando expostas à luz ultravioleta, B1 e B2 azul e G1 e G2 verde (HARTLEY et al., 1963; NESBITT et al., 1962). Devido à possibilidade de ocorrerem falsos positivos, a confirmação do resultado obtido foi feita pela cromatografia bidimensional, onde os interferentes são eliminados e as aflatoxinas presentes na amostra podem ser observadas na diagonal da placa, pois elas se movem na mesma distância em ambas as direções (Figura 1). A placa bidimensional confirmou a presença das aflatoxinas B1(AFB1), B2 (AFB2), G1 (AFG1) e G2 (AFG2) no farelo de algodão (Figura 1), sendo que a concentração total de aflatoxinas encontrada na amostra foi de $76,4 \mathrm{ng} . \mathrm{g}^{-1}$, correspondente a 43,5ng. ${ }^{-1}$ de AFB 1; 15,2ng.g ${ }^{-1}$ de AFB2; 9,1ng. g $^{-1}$ de AFG1 e 8,6ng.g ${ }^{-1}$ de AFG2, acima do permitido pela legislação vigente $\left(50 \mathrm{ng}^{-\mathrm{g}^{-1}}\right.$ soma das 4 aflatoxinas).

Foi detectada aflatoxina M1 na concentração $\geq 0,5 \mathrm{ng} \cdot \mathrm{mL}^{-1}$ pelo ensaio imuno-cromatográfico e quantificada na concentração de $0,64 n g \cdot \mathrm{mL}^{-1}$ por CLAE, com o tempo de retenção de 4,025 minutos para a amostra (Figura 2) e 4,206 minutos para o padrão.

As concentrações de aflatoxinas encontradas no farelo de algodão tiveram como conseqüência a queda da produção de leite de $14 \mathrm{~L}$ dia $^{-1}$ para 11L.dia ${ }^{-1}$ durante os 6 meses em que foi utilizado como complemento. Outra conseqüência bastante séria foi a contaminação do leite, que foi comercializado e consumido pelos empregados da fazenda, incluindo crianças, mas não foi relatado nenhum quadro de intoxicação aguda, entretanto a intoxicação crônica não pode ser descartada. 


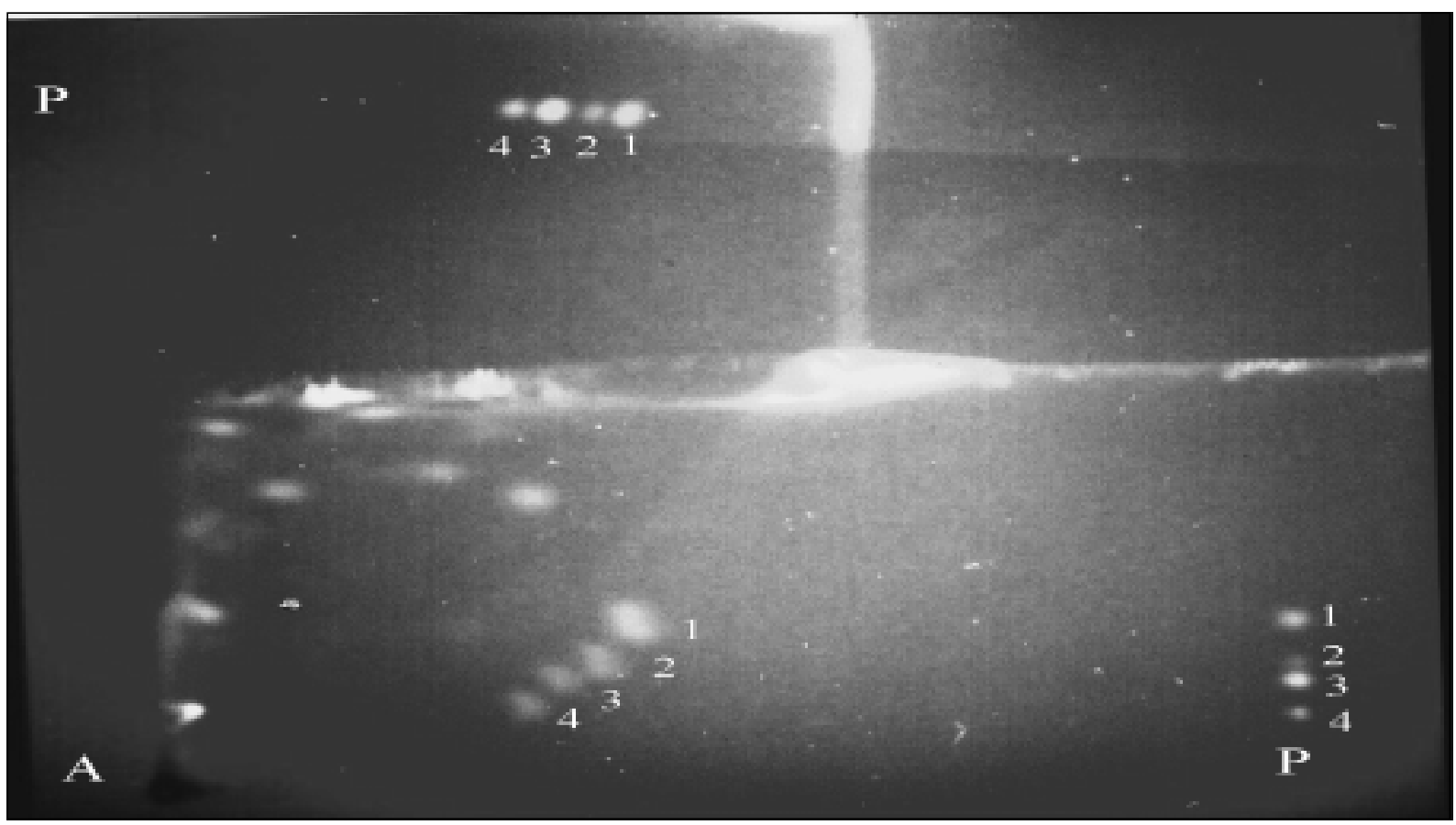

Figura 1 - Placa bidimensional. $\mathrm{P}$ = ponto de aplicação do padrão de aflatoxinas B1, B2, G1 e G2; A= ponto de aplicação da amostra; $1=$ aflatoxina B1; 2= aflatoxina B2; 3 = aflatoxina G1 e 4 = aflatoxina G2.

Semente de algodão, seus derivados e milho são considerados por autoridades americanas como os produtos de maior risco de contaminação por aflatoxinas utilizados na alimentação de vacas lactantes, aumentando o risco de contaminação do leite (APPLEBAUM et al., 1982), o que levou Estados e Federação restringirem a concentração de aflatoxinas para níveis inferiores a $20 \mu \mathrm{g} . \mathrm{g}^{-1}$ nestes produtos (PRICE et al., 1985).

Para prevenir a contaminação do leite com AFM1 em níveis inferiores a $0,5 \mu \mathrm{g} . \mathrm{L}^{-1}$, é necessário estabelecer a taxa de conversão de aflatoxinas em alimentos naturalmente contaminados, pois a quantidade de AFM1 excretada pelo leite é diretamente proporcional à quantidade de AFB1 ingerida, sendo detectada no leite dois dias após a ingestão e desaparecendo 3 a 4 dias após a retirada da AFB 1 da dieta (APPLEBAUM et al., 1982; FROBISH et al., 1986; PRICE et al., 1985).

Boas práticas agrícolas, de transporte, de manufatura e de armazenagem continuam sendo a melhor forma de prevenir a contaminação de alimentos por aflatoxinas e, conseqüientemente a contaminação do leite. Assim, estratégias e instrumentos legais são necessários na agricultura e na indústria de alimentos

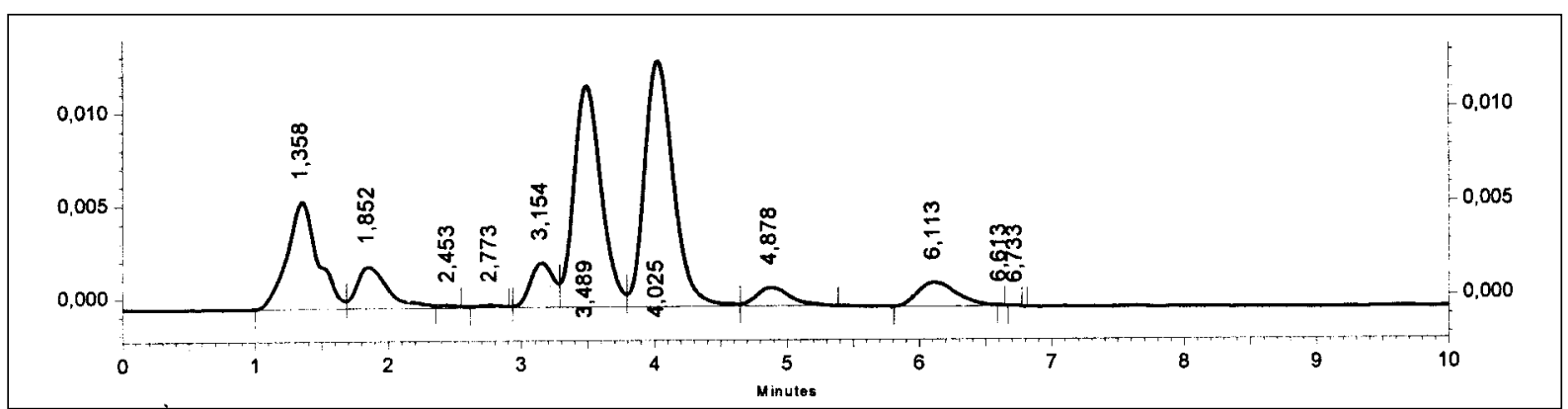

Figura 2 - Cromatograma da amostra de leite, no qual a aflatoxina M1 corresponde ao pico com tempo de retenção 4,025 minutos com concentração de $0,64 \mathrm{ng} / \mu \mathrm{L}$. 


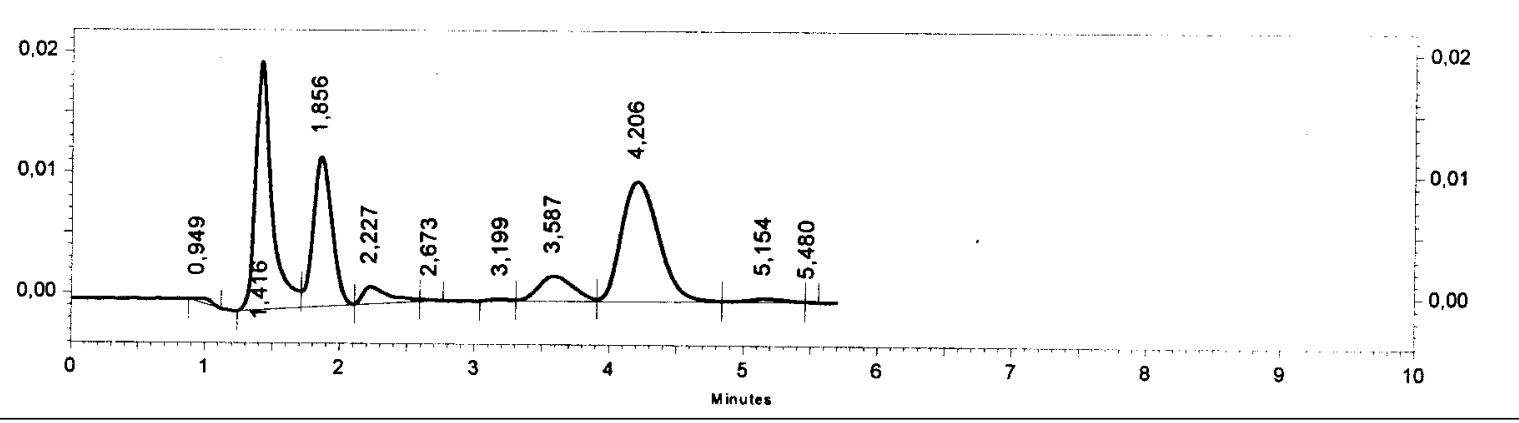

Figura 3 - Cromatograma do padrão de aflatoxina M1, correspondendo ao pico com tempo de retenção 4,206 minutos com concentração de $0,322 \mathrm{ng} / \mu \mathrm{L}$.

para assegurar a qualidade de produtos de origem animal.

\section{FONTESDE AQUISIÇÃO}

${ }^{1}$ Padrão de aflatoxinas - Sigma-Aldrich - Saint Louis, Missouri / USA

${ }^{2}$ Cromatofolha 1.05553 - Merck - Darmstadt / Alemanha ${ }^{3}$ Fotodensitômetro CS 9000 - Shimadzu - Kyoto / Japão ${ }^{4}$ Tira-teste Charm SL Aflatoxin Test - Charm Sciences Inc USA

${ }^{5}$ Coluna de imunoafinidade - Vicam - USA

${ }^{6}$ Cromatógrafo líquido de alta eficiência LC 6 AD - Shimadzu

- Kyoto / Japão

\section{REFERÊNCIAS BIBLIOGRÁFICAS}

APPLEBAUM, R.S. et al. Aflatoxin: toxicity to dairy cattle and occurence in milk and milk products - a review. Journal of Food Protection, v.45, n.8, p.752-777, 1982.

AOAC 970.45. Official Methods of Analysis of AOAC. $15^{\text {th }}$ ed. Arlington, VA : Association of Official Analytical Chemists, 1990. p.49.10.

AOAC 985.17. Official Methods of Analysis of AOAC. $15^{\text {th }}$ ed. Arlington, VA : Association of Official Analytical Chemists, 1990. p.49.28-49.29.

DRAGACCI, S.; GROSSO, F.; GILBERT, J. Immunoaffinity column clean-up with liquid chromatography for determination of aflatoxin M1 in liquid milk. Journal AOAC International, v.82, n.2, p.437-443, 2001.

FROBISH, R.A. et al. Aflatoxin residues en milk of dairy cows after ingestion of naturally contaminated grain. Journal of Food Protection, v.49, n.10, p.781-785, 1986.

HARTLEY, R.D.; O'KELLY, J. Toxicity and fluorescence properties of aflatoxins. Nature, v.196, p. $1001,1963$.

HUSSEIN, S.H.; BRASEL, J.M. Toxicity, metabolism, and impact of mycotoxins on humans and animals. Toxicology, v.167, p.101-134, 2001.

LÓPEZ, C. et al. Distribution of aflatoxin M1 in cheese obtained from milk artificially contaminated. International Journal of Food Microbiology, v. 64, p. $211-215,2001$.

NESBITT, B.F. et al. Toxic metabolites of Aspergillus flavus. Nature, v.195, p.1062-1063, 1962.

PRICE, L. R. et al. Aflatoxin conversion by dairy cattle consuming naturally-contaminated whole cottonseed. Journal of Food Protection, v.48, n.1, p.11-15, 1985.

SHARMA, R.P.; SALUNKHE, D.K. Introduction to mycotoxins. In: SHARMA, R.P. et al. Mycotoxins and Phytoalexins. London : CRC, 1991. p.3-12.

STEINHART C.E.; DOYLE M.E.; COCHRANE B.A. Food Safety 1996. New York : Marcel Dekker, 1996. p.376-394. 